\title{
Association of Increased Arterial Stiffness with Severity of Coronary Artery Disease in Patients with Non-ST- Segment Elevation Myocardial Infarction
}

\author{
A.B.M. Nizam Uddin ${ }^{1}$, Abdullah-Al- Shafi Majumder², Md. Abdul Kader Akanda², Shahana Zaman ${ }^{3}$, Mohammad \\ Anowar Hossain ${ }^{1}$, Ashiqur Rahman Khan ${ }^{1}$, Muhammed Aminur Razzaque ${ }^{1}$, Pinaki Ranjan Das ${ }^{1}$, Md. Zahidul \\ Islam ${ }^{1}$, Kazi Ashraful Alam ${ }^{4}$
}

\begin{abstract}
:
Objective: Cardiovascular diseases (CVDs) have become the single largest cause of death worldwide. The scenario is same in Bangladesh. Increased arterial stiffness has been associated with increased risk of CVD. A noninvasive assessment of arterial stiffness may serve as a useful adjunct to the cardiovascular risk stratification and risk management and it would be of value for the examination of larger populations. So, this study was designed to demonstrate the association between CVD and arterial stiffness.

Methods: This cross sectional study was conducted in the National Institute of Cardiovascular Disease, Dhaka over a period of ten months starting from January 2014. Patients were purposively selected from those who were admitted in NICVD with acute Non-ST- Segment Elevation Myocardial Infarction (NSTEMI). A total of $\mathbf{8 0}$ patients were included in the study. Study patients were
\end{abstract}

divided into two groups on the basis of aortic augmentation pressure (AP), augmentation index (Aix) and augmentation index corrected at heart rate $75 / \mathrm{min}$ (Alx@75). Assessment of coronary angiography was performed and severity was assessed by several scoring systems.

Results: Significant positive correlation was found between Alx@75 and vessel score ( $r=0.70, p=0.001)$, Friesinger score $(r=0.66, p=0.001)$ and Leaman score $(r=$ $0.69, p=0.001)$. Logistic regression analysis showed that increasedAlx@75 is significantly associated with severe Coronary Artery Disease (CAD) (OR being 5.54).

Conclusion: The results indicate that AP, Alx and Alx@75 are predictors of severity of CAD. It may be considered as a recommended test for the evaluation of cardiovascular risk.

Keywords: Arterial Stiffness; Coronary artery disease; Myocardial infarction

(Bangladesh Heart Journal 2019; 34(2): 73-79)

1. Assistant Registrar, Dept. of Cardiology, National Institute of Cardiovascular Diseases\& Hospital, Sher-e-Bangla Nagar, Dhaka, Bangladesh.

2. Professor, Department of Cardiology, National Institute of Cardiovascular Diseases\& Hospital, Sher-e-Bangla Nagar, Dhaka, Bangladesh.

3. Registrar, Depat. of Cardiology, National Institute of Cardiovascular Diseases\& Hospital, Sher-e-Bangla Nagar, Dhaka, Bangladesh

4. Medical Officer, Dept. of Cardiology, National Institute of Cardiovascular Diseases\& Hospital, Sher-e-Bangla Nagar, Dhaka, Bangladesh.

Address of Correspondence : A. B. M. Nizam Uddin, Assistant Registrar, Department of Cardiology, National Institute of Cardiovascular Diseases, Sher-e-Bangla Nagar, Dhaka, Bangladesh. Email: dr.nizam@mail.com, Contact no: +8801716245208
Introduction:

Cardiovascular diseases (CVDs) have become the single most cause of death worldwide over the last decade. During the last century, low- and middle-income countries has been developed an alarming increase in the rates of CVD like the high-income countries ${ }^{1}$.

An estimated 17.3 million people died worldwide from CVDs in 2008, representing $30 \%$ of all global deaths. By 2030, 23.3 million people will die from CVDs, mainly from heart disease and stroke ${ }^{2}$. In a WHO 2008 estimate, there were 834.4 deaths per 100,000 population due to CVDs and diabetes mellitus in Bangladesh².

DOI: https://doi.org/10.3329/bhj.v34i2. 44436

Copyright (C 2017 Bangladesh Cardiac Society. Published by Bangladesh Cardiac Society. This is an Open Access articles published under the Creative Commons Attribution-NonCommercial 4.0 International License (CC BY-NC). This license permits use, distribution and reproduction in any medium, provided the original work is properly cited and is not used for commercial purposes. 
The prevalence of coronary artery disease (CAD) was estimated as 3.3 per thousand in 1976 and 17.2 per thousand in 1986 indicating fivefold increase of the disease by ten years ${ }^{3}$.It is well known $t \quad h \quad a \quad t$ smoking, hypertension, diabetes mellitus, dyslipidemia, family history of premature CAD and obesity are most important risk factors for $\mathrm{CAD}^{4}$.

Risk is an important driver of management decisions. Accurate but simple methods of risk assessment are important for patient care, determining the prognosis and providing information for the patient ${ }^{5}$.

Despite improvement in risk scoring, there still remain patients identified as being low risk who experience CAD events, as well as, patients deemed high risk who remain free of CAD events. This has led to a search for additional emerging risk variables ${ }^{6}$.

Endothelium modulates arterial stiffness, which precedes overt atherosclerosis and is an independent predictor of cardiovascular events. Dysfunction of the endothelium may be considered as an early and potentially reversible step in the process of atherogenesis. As the atherosclerosis progresses, the tunica media thickens and the tunica intima becomes rigid, and this reduces the arterial elasticity ${ }^{7}$.

Increased arterial stiffness has been associated with increased risk of $\mathrm{MI}$, stroke, congestive heart failure and overall morality ${ }^{8}$. Increased arterial stiffness correlates with coronary risk factors as well as measures of arterial stiffness correlate with the presence of angiographic CAD ${ }^{9}$.

Arterial stiffness determined invasively, has been shown to predict a higher risk of coronary atherosclerosis. However, invasive techniques are of limited value for screening and risk stratification in larger patient groups. Therefore, a noninvasive assessment of arterial stiffness may serve as a useful adjunct to the cardiovascular risk stratification and risk management ${ }^{10}$.

The central aortic pressure wave is composed of a forward-traveling wave generated by left ventricular ejection and a later-arriving reflected wave from the periphery ${ }^{11}$. As aortic and arterial stiffness increase, transmission velocity of both forward and reflected waves increase, which causes the reflected wave to arrive earlier in the central aorta and augment pressure in late systole. Therefore, augmentation of the central aortic pressure wave is a manifestation of early wave reflection and is the boost of pressure from the first systolic shoulder to the systolic pressure peak ${ }^{12}$ can be expressed in absolute terms (augmented pressure [AP]) or as a percentage of pulse pressure (augmentation index [Alx]). Alx, determined noninvasively, has been shown to be predictive for coronary artery disease (CAD) ${ }^{9}$.

Recently done three studies showed significant association of CAD severity with arterial stiffness measured by non invasive pulse wave velocity (PWV) and pulse wave analysis (PWA) method ${ }^{13-15}$.

While there are several studies from developed countries on the severity of CAD and arterial stiffness in AMI patients, there are not enough data from Bangladesh addressing PWA as an independent risk assessment tool for detecting severity of CAD.

Because a noninvasive approach clearly would be of value for the examination of larger populations, this observational study was designed to demonstrate in detail the association between CAD and aortic Alx, assessed by noninvasive PWA.

\section{Materials and methods:}

This cross sectional study was conducted in the National Institute of Cardiovascular Disease (NICVD), Dhaka over a period of ten months starting from January 2014 to October 2014. Patients were purposively selected from those who were admitted in NICVD with NSTEMI agreed to do coronary angiography. Total 80 patients (male: 66, female: 14) were included in this study. Assessment of arterial stiffness was performed noninvasively with the commercially available SphygmoCor system (The SphygmoCor Vx PWA system Model SCOR-Mx DCN: 100521 P/N:1- 00418, Rev:9.0/0-0m, SphygmoCor Software Version: 8, AtCor Medical Private Ltd) using applanation tonometry with a high-fidelity micromanometer (Millar Instruments). Patients with valvular, congenital heart disease and cardiomyopathy, suspected myocarditis or pericarditis, major non cardiovascular disorder such as severe renal impairment, uncontrolled hypertension (systolic blood pressure $>160 \mathrm{mmHg}$ ), prior $\mathrm{PCl}$ or CABG were excluded from the study. From where study patients were divided into two groups on the basis of aortic augmentation pressure (AP), augmentation index $(A \mid x)$ and augmentation index corrected at heart rate $75 / \mathrm{min}$ (Alx@75). 40 patients AP, Alx and Alx@75 were normal (Group I) and 40 patients AP, Alx and Alx@75 were increased (Group II). Informed consent was obtained in accordance with the study protocol approved by the local ethical committee.

NSTEMI was diagnosed by third universal definition of Myocardial Infarction ${ }^{16}$. Peripheral pressure waveforms were recorded from the radial artery at the wrist, sing 
applanation tonometry. After 20 sequential waveforms were acquired, a validated generalized transfer function was used to generate the corresponding central aortic pressure waveform. Alx and AP were derived from this with the technique of PWA. The Alx was defined as the AP divided by pulse pressure and expressed as a percentage. Larger values of Alx will indicate increased wave reflection from the periphery or earlier return of the reflected wave as a result of increased PWV (attributable to increased arterial stiffness) and vice versa. Because Alx was influenced by heart rate Alx@75 was used. Only high-quality measurements was taken in the sitting position in a quiet, temperature-controlled room $\left(22 \pm 1^{\circ} \mathrm{C}\right)$ after a brief period (at least 5 minutes) of rest, most often on the day before cardiac catheterization by a doctor not involved in performance or interpretation of the angiograms. Coronary angiography was performed by percutaneous femoral approach using standard angiographic techniques. Interpretation of coronary angiogram was done by visual estimation by two cardiologists. Severity of coronary stenosis was assessed by Vessel score, Friesinger score and Leaman score. Friesinger score 5 points was regarded as cut-off value for CAD severity (Friesinger score $<5$ points insignificant CAD, Friesinger score e" 5 points - significant CAD) $)^{17 .}$

The numerical data obtained from the study was analyzed and significance differences was estimated by using statistical methods. Data were presented as frequency and percents for categorical variables and as mean with standard deviation for quantitative variables. Categorical variables were analyzed by chi-square test. Quantitative variables were analyzed by t-test or ANOVA. Correlation between Alx@75 and angiographic severity was measured by Spearman's correlation test. $\mathrm{P}<0.05$ was considered as significant. Statistical analyses were performed with SPSS, version 16.0 (SPSS Inc).

\section{Results:}

Majority of the study patients belonged to 41-50 and 5160 years age in both groups. The mean age was found 49.6 \pm 8.5 years in Group I and $51.4 \pm 7.3$ years in Group II. Male female ratio was 4.7:1. Among the risk factors hypertension, diabetes mellitus and family history of CAD were significantly higher in group II than group I $(p=0.01$, $0.02,0.03)$. Higher the number of vessels involved, the greater is the Alx@75 with mean score in single, double and triple vessel disease being 14.43, 20.43 and 30.68 respectively. The mean vessel score for group II patients was $2.58 \pm 0.71$ and that of group I patients was $0.98 \pm 0.73$ and the difference was statistically significant $(p=0.001)$. Low Friesinger score was greater in group I with statistically significant difference $(p=0.001)$. High Friesinger score was significantly higher in group II. $(p=0.001)$.The mean AP, Alx and Alx@75 in significant CAD was $9.13 \pm 5.28 \%, 27.91 \pm 14.42 \%$ and $27.85 \pm 11.51$ and insignificant CAD was $4.85 \pm 4.27,14.67 \pm 10.68$ and $13.30 \pm 8.43$. The differences were statistically significant $(p<0.05)$. There is a positive correlation between Alx@75 and CAD severity in terms of vessel score $(r=0.70)$, Friesinger score $(r=0.66, p=0.001)$ and Leaman Score $(r=0.69)$. Logistic regression analysis showed that patient with increased Alx@75 had 5.54 times higher risk of having significant CAD compared with those with the normal Alx@75.

Table-I

Demographic profile of study population $(n=80)$

\begin{tabular}{|c|c|c|c|}
\hline & Group $1(n=40)$ & Group II $(n=40)$ & $P$ value \\
\hline Age in years (Mean $\pm S D)$ & $49.6 \pm 8.5$ & $51.4 \pm 7.3$ & $0.30^{\text {ns }}$ \\
\hline \multicolumn{4}{|l|}{ Gender } \\
\hline Male & $33(82.5 \%)$ & $33(82.5 \%)$ & $1.00^{\mathrm{ns}}$ \\
\hline Female & $7(17.5 \%)$ & $7(17.5 \%)$ & $1.00^{\mathrm{ns}}$ \\
\hline \multicolumn{4}{|l|}{ Risk Factors } \\
\hline Smoking & $18(45 \%)$ & $22(55 \%)$ & $0.37^{\mathrm{ns}}$ \\
\hline Hypertension & $6(15 \%)$ & $16(40 \%)$ & $0.01^{\mathrm{s}}$ \\
\hline Diabetes Mellitus & $6(15 \%)$ & $15(37.5 \%)$ & $0.02^{\mathrm{s}}$ \\
\hline Dyslipidaemia & $8(20 \%)$ & $9(22.5 \%)$ & $0.78^{\text {ns }}$ \\
\hline Family $\mathrm{H} / \mathrm{O}$ of CAD & $9(22.5 \%)$ & $18(45 \%)$ & $0.03^{s}$ \\
\hline
\end{tabular}

s-significant, ns-not significant. 
Table-II

Distribution of the study patients according to vessel score $(n=80)$

\begin{tabular}{|c|c|c|c|c|c|}
\hline \multirow[t]{2}{*}{ Vessel Score } & \multicolumn{2}{|c|}{ Group I $(n=40)$} & \multicolumn{2}{|c|}{ Group II $(n=40)$} & \multirow[t]{2}{*}{$P$ value } \\
\hline & Number & $\%$ & Number & $\%$ & \\
\hline Score - 0 & 10 & 25.0 & 1 & 2.5 & $0.003^{s}$ \\
\hline Score - 1 & 22 & 55.0 & 2 & 5.0 & $0.001^{\mathrm{s}}$ \\
\hline Score - 2 & 7 & 17.5 & 10 & 25.0 & $0.41^{\mathrm{ns}}$ \\
\hline Score - 3 & 1 & 2.5 & 27 & 67.5 & $0.001^{\mathrm{s}}$ \\
\hline Mean $\pm S D$ & \multicolumn{2}{|c|}{$0.98 \pm 0.73$} & \multicolumn{2}{|c|}{$2.58 \pm 0.71$} & $0.001^{\mathrm{s}}$ \\
\hline
\end{tabular}

s-significant, ns-not significant.

Table-III

Association between Alx@75 and number of vessels involved ( $n=80)$

\begin{tabular}{lccc}
\hline No. of vessel involved & \multicolumn{2}{c}{ Alx@75 } & P value \\
\cline { 2 - 3 } & Mean & SD & $0.001^{\mathrm{s}}$ \\
\hline None $(n=7)$ & 13.14 & 8.78 & \\
Single $(n=21)$ & 14.43 & 8.58 & 13.91 \\
Double $(n=15)$ & 20.33 & 9.67 & \\
Triple $(n=37)$ & 30.68 & & \\
\hline
\end{tabular}

s-significant, ns-not significant.

Table-IV

Distribution of the study patients according to Friesinger score $(n=80)$

\begin{tabular}{|c|c|c|c|c|c|}
\hline \multirow[t]{2}{*}{ Friesinger Score } & \multicolumn{2}{|c|}{ Group I $(n=40)$} & \multicolumn{2}{|c|}{ Group II $(n=40)$} & \multirow[t]{2}{*}{$\mathrm{P}$ value } \\
\hline & Number & $\%$ & Number & $\%$ & \\
\hline Normal (0) & 4 & 10.0 & 1 & 2.5 & $0.18^{\text {ns }}$ \\
\hline Low $(1-4)$ & 21 & 52.5 & 1 & 2.5 & $0.001^{\mathrm{s}}$ \\
\hline Intermediate $(5-10)$ & 14 & 35.0 & 12 & 30.0 & $0.63^{\mathrm{ns}}$ \\
\hline High $(11-15)$ & 1 & 2.5 & 26 & 65.0 & $0.001^{\mathrm{s}}$ \\
\hline
\end{tabular}

s-significant, ns-not significant.

Table-V

Mean status of $A P, A l x$ and $A / x @ 75 b p m$ of the study patients according to significant coronary artery disease defined by Friesinger score $(n=80)$

\begin{tabular}{lccc}
\hline Variables & $\begin{array}{c}\text { Significant CAD } \\
\text { (Friesinger score } \geq 5)\end{array}$ & $\begin{array}{c}\text { Insignificant CAD } \\
(\text { Friesinger score 0-4) }\end{array}$ & $\begin{array}{c}\text { Palue } \\
\text { Mean } \pm \text { SD }\end{array}$ \\
\hline AP (\%) & $9.13 \pm 5.28$ & $4.85 \pm 4.27$ & $0.001^{\mathrm{s}}$ \\
Alx (\%) & $27.91 \pm 14.42$ & $14.67 \pm 10.68$ & $0.001^{\mathrm{s}}$ \\
Alx@75 (\%) & $27.85 \pm 11.51$ & $13.30 \pm 8.43$ & $0.001^{\mathrm{s}}$ \\
\hline
\end{tabular}

s-significant, ns-not significant. 


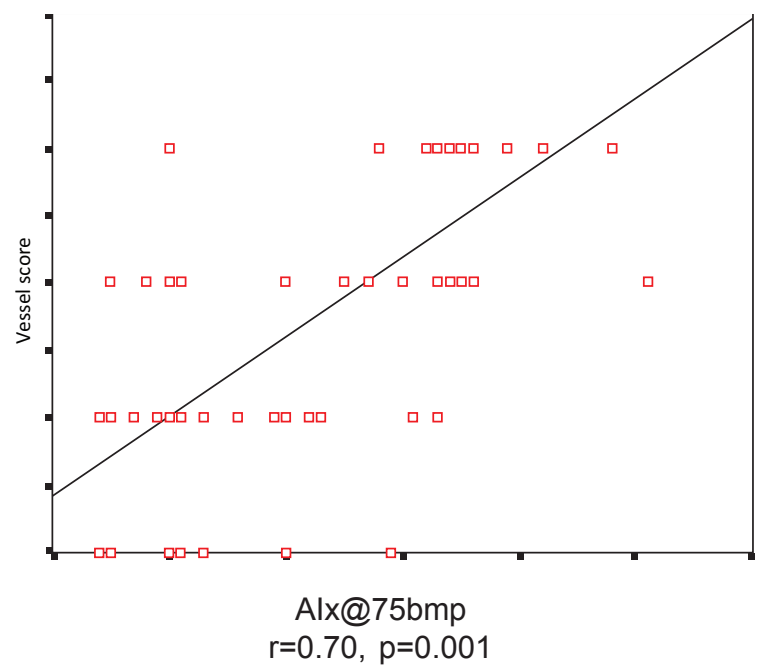

Fig.-1: Correlation between Aix@75 and vessel score

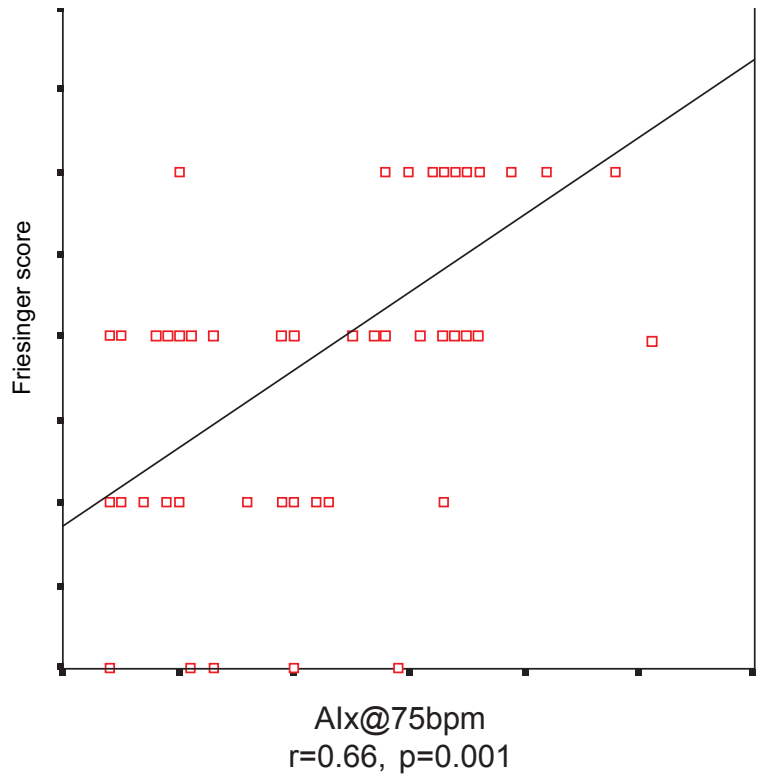

Fig.-2: Correlation between Aix@75 and Friesinger score

Table- V

Multivariate logistic regression of determinants of CAD (by Friesinger Score).

\begin{tabular}{lccccc}
\hline Variables of interest &, & S.E. & P value & OR & $95 \% \mathrm{Cl}$ \\
\hline Smoking & 0.301 & 0.230 & $0.41^{\mathrm{NS}}$ & 1.08 & $0.516-2.201$ \\
Hypertension & 0.416 & 0.243 & $0.08^{\mathrm{NS}}$ & 1.45 & $0.415-3.934$ \\
Diabetes mellitus & 0.689 & 0.543 & $0.04^{\mathrm{S}}$ & 1.71 & $1.187-3.784$ \\
Increased TG & 0.120 & 0.241 & $0.11^{\mathrm{NS}}$ & 0.88 & $0.555-2.919$ \\
Family history of CAD & 0.579 & 0.418 & $0.02^{\mathrm{S}}$ & 2.97 & $1.257-5.778$ \\
Increased Alx@75 & 1.252 & 0.801 & $0.01^{\mathrm{S}}$ & 5.54 & $1.824-10.201$ \\
\hline
\end{tabular}

Dependent variable: CAD; independent variables; smoking, hypertension, diabetes mellitus, increased TG, positive family history of CAD and increased Alx@75.

$\mathrm{S}=$ Significant, NS= Not significant.

\section{Discussion:}

The mean age of group I was $49.6 \pm 8.5$ years and in group II was $51.4 \pm 7.3$ years. The mean age of group II was higher than group I but the difference not statistically significant $(p=0.30)$.

Male female ratio was 4.7:1. Similar male preponderance was found in almost all studies relating to $\mathrm{IHD}^{13-15}$.

In group I the mean level of Alx@75 was observed $10.42 \pm 5.74 \%$ in male and $17.71 \pm 4.75 \%$ in female and in group II $32.67 \pm 2.89 \%$ in male and $41.29 \pm 6.21 \%$ in female. So Alx@75 is significantly higher in female than male. Khan, et al. ${ }^{13}$ and Gatzka, et al. ${ }^{18}$ showed significantly higher Alx@75 in female than male.
The distribution of common risk factors for CAD in the present study revealed that smoking habit was found in $50 \%$ followed by positive family history of premature CAD $33.8 \%$, hypertension $27.5 \%$, diabetes mellitus $26.2 \%$, dyslipidaemia $21.2 \%$. Khan, et al. ${ }^{13}$ had shown that smoking was the highest prevalent risk factor.

The mean central systolic blood pressure was $115.5 \pm 17.2 \mathrm{mmHg}$ in group I and $105.4 \pm 20.2 \mathrm{mmHg}$ in group II.

The mean central diastolic blood pressure was $86.9 \pm 12.9 \mathrm{mmHg}$ in group I and $75.8 \pm 9.9 \mathrm{mmHg}$ in group II. The mean central pulse pressure was $27.3 \pm 8.7 \mathrm{mmHg}$ in group I and $32.6 \pm 8.1 \mathrm{mmHg}$ in group II. The mean 
difference of the above statistic were statistically significant $(p<0.05)$.

Uddin ${ }^{19}$ showed increased central and brachial pulse pressure and systolic pressure was significantly associated with CAD severity.

Uddin ${ }^{19}$ also found that compared to brachial systolic and pulse pressure, central aortic pulse pressure appears to be a better predictor of the presence and extent of coronary atherosclerosis which was similar to the findings of the present study.

In this study it was found that among group II, highest percentage was of 3 vessel score $(67.5 \%)$ and 2 vessel score $(25 \%)$ followed by 1 vessel score $(05 \%)$. On the contrary among group I, highest percentage was of 1 vessel score (55\%). $17.5 \%$ and $2.5 \%$ belonged to 2 and 3 vessel score and $25 \%$ patient had 0 vessel score.

The mean vessel score for group II patients was $2.58 \pm 0.71$ and that of group I patients was $0.98 \pm 0.73$ and the mean difference was statistically significant $(p=0.001)$.

The mean Alx@75 of subjects with normal angiographic findings was $13.14 \pm 8.78 \%$. The mean Alx@75 of single, double and triple vessel disease were $14.43 \pm 8.58$, $20.33 \pm 13.91$ and $30.68 \pm 9.67$ (\%) respectively.

The Alx@75 increased in proportion with the number of vessels involved and the differences were statistically significant $(p=0.001)$. Khan, et al. ${ }^{13}$ and Das ${ }^{15}$ also found that Alx@75 was increased in proportion with the number of vessels involved. Park, et al. ${ }^{20}$ and Weber, et al. ${ }^{21}$ also found that arterial stiffness index increased significantly with the increasing number of stenotic coronary vessels.

In the present study, analysis of Friesinger score revealed that normal Friesinger score (0) was found in 2.5\% patients in group II and $10 \%$ patients in group I. Low Friesinger score (1-4) was found in $2.5 \%$ and $52.5 \%$ patients in group II and group I respectively. High Friesinger score (11-15) was found in $65 \%$ patients in group II and $2.5 \%$ patients in group I.

Low Friesinger score was significantly higher in Group I $(p=0.001)$. High Friesinger score was significantly higher in group II. $(p=0.001)$.

Khan, et al. ${ }^{13}$ and Das. ${ }^{15}$ also found that high Friesinger score was higher among patients with increased arterial stiffness.

The mean Alx@75 was observed 27.85 $111.51 \%$ and $13.30 \pm 8.43 \%$ in significant and insignificant $C A D$ respectively and the difference was statistically significant $(p=0.001)$. Weber, et al. ${ }^{21}$ found that mean Alx@75 was observed $16.9 \pm 9.9 \%$ and $13.4 \pm 10.6 \%$ in significant and insignificant $C A D$ respectively and the difference was statistically significant $(p=0.01)$.

In this study there was a positive correlation between Alx@75 and CAD severity in terms of vessel score, Friesinger score and Leaman score $(r=0.70, r=0.66$ and $r=0.69$ respectively, $p=0.001$ ).

Khan, et al. ${ }^{13}$ and Das. ${ }^{15}$ also showed positive correlation between arterial stiffness and and CAD severity in terms of vessel score, Friesinger score and Leaman score.

The variables revealed to be significantly associated with severe CAD by multivariate analysis were increased Alx@75, positive family history of CAD and diabetes mellitus with ORs being 5.54, 2.97 and 1.71 respectively.

\section{Conclusion:}

The results presented here indicates that AP, Alx and Alx@75 measures closely related to arterial stiffness, are predictors of severity of CAD. It may be considered as recommended test for the evaluation of cardiovascular risk in addition to other routine investigation.

\section{Limitations:}

This was non-randomized study. The sample size was small and the study was carried out in one centre.

Angiography was evaluated by visual estimation, so there was chance of inter observer and intra observer variation of interpretation of the severity of the stenosis.

\section{Disclosure:}

This research project was self funded and was not by any group or any institution.

\section{References:}

1. Gaziano TA. Gaziano, JM. Global burden of cardiovascular disease. In: Bonow RO, Mann DL, Zipes, DP,LibbyP, editors. Braunwald's Heart disease: a text book of cardiovascular medicine. St. Louis: Elsevier; 2012. 1-20.

2. World Health Organization. WHO Cardiovascular Disease. Factsheet No 317. 2011. Available from: http://www.who.int/mediacentr e/factsheets/fs317/ en/index.ht $\mathrm{ml}$.

3. Islam MN, Ali MA, Ali M. Spectrum of Cardiovascular Disease: The current scenario in Bangladesh. Bangladesh Heart Journal. 2004; 19: 1-7.

4. Reddy KS, Yousuf S.Emerging epidemic of cardiovascular disease in developing countries. Circulation. 1998; 97: 596-601. 
4. Topol EJ, Lauer MS. The rudimentary phase of personalized medicine: coronary risk scores. Lancet. 2003; 362:1776-1777.

5. David J, Maron PM, Ridoker. Preventive strategies for coronary heart disease. In: Fuster V, O'Rouke R, Walsh RA, Poole-Wilson P, editors. Hurst's The Heart. New York: The Mc Graw-Hill; 2011. 1203-1234.

6. Vanhoutte PM. Endothelial control of vasomotor function: From health to coronary disease. Circulation. 2003; 67:572-575.

7. Oliver JJ, Webb DJ. Noninvasive Assessment of Arterial Stiffness and Risk of Atherosclerotic Events. Arterioscler Throm Vasc Biol. 2003; 23:554566.

9. Hayashi T, Nakayama Y, Tsumurak M, Yoshimaru K. Reflection in the arterial system and the risk of coronary heart disease. Am J Hypertens. 2002; 15:415-419.

10. Madhavan S, Ooi WL, Cohen H, Alderman MH. Relation of pulse pressure and blood pressure reduction to the incidence of myocardial infarction. Hypertension. 1994; 23:395-401.

11. Nichols WW, Singh BM. Augmentation index as a measure of peripheral vascular disease state. Curr Opin Cardiol. 2002; 17:543-551.

12. O'Rourke MF, Mancia G. (1999). Arterial stiffness. J Hypertens. 1999; 17:1-4.

13. Khan AR, Majumder AAS, Rahman MT, Hoque M. Increased arterial stiffness correlates with the severity of coronary artery disease in patients with acute ST elevation myocardial infarction. Cardiovascular Journal. 2013; 5:133-140.
14. Razzaque MA. Association of aortic pulse wave velocity with the severity of coronary artery disease in patients with acute ST elevation myocardial infarction [Thesis]. University of Dhaka; 2013.

15. Das PR. Association of aortic pulse wave velocity with the severity of coronary artery disease in patients with non- ST-segment elevation myocardial infarction. [Thesis]. University of Dhaka; 2014.

16. Thygesen K, Alpert JS, Jaffe AS, Simmons ML, Chaitman BR, White HD. Third universal definition of myocardial infarction. Circulation. 2012;126: 2020-35.

17. Ringqvist I, Fisher LD, Mock M, Devis KB. Prognostic value of Angiographic Indices of coronary artery disease from the Coronary Artery Surgery Study (CASS). J Clin Invest. 1983; 71:1854-1866.

18. Gatzka CD, Kingwell BA, Cameron JD. Gender differences in the timing of arterial wave reflection beyond differences in body height. J Hypertens. 2001; 19:2197-2203.

19. Uddin MM. Association of central pulse pressure with the extent of angiographically detected coronary artery disease [Thesis], Dhaka Medical College, Dhaka. 2011.

20. Park SM, Seo HS, Lim HE, Shin HS, Park CG, Oh DJ, et al. Assessment of the Arterial Stiffness Index as a Clinical Parameter for Atherosclerotic Coronary Artery Disease. Korean circ J. 2004; 34(7):677-683.

21. Weber T, Auer J, O’Rourke MH. Arterial stiffness, wave reflections and the risk of coronary artery disease. Circulation. 2004; 109:184-189. 